\title{
Exploratory Completeness Analysis of Mapillary for Selected Cities in Germany and Austria
}

\author{
Levente Juhasz and Hartwig Hochmair \\ University of Florida, Fort Lauderdale/USA·levente.juhasz@ufl.edu \\ Full paper double blind review
}

\begin{abstract}
Mapillary, founded in early 2014, is a Web 2.0 application that allows voluntary users to contribute street level photographs from all over the world. This paper analyses the growth in uploaded data and contributor numbers over time, and assesses data completeness by comparing Mapillary with Google Street View and OpenStreetMap data for selected cities in Germany and Austria. Results show that as of now Google Street View generally provides a better coverage in the cities in which it is offered, but that Mapillary has the potential to reach higher coverage along off-road segments, such as footpaths and bicycle trails, or even railroads.
\end{abstract}

\section{Introduction}

Street level photographs are an important data source for a variety of analysis tasks, including the identification of road features (e.g., crosswalks, traffic signs) and the assessment of wheelchair accessibility of sidewalks. Besides commercial products such as Google Street View or Mapjack, Mapillary is the first platform offering a street level photograph service based on crowd-sourced data. It therefore provides a unique addition to the currently available data resources of Volunteered Geographic Information (VGI) (GoODCHILD 2007). Mapillary is run by a company located in Malmö, Sweden, and began providing services at the beginning of 2014. Imagery is available (at http://www.mapillary.com) to the public for any kind of purpose under an open license, CC BY-SA 4.0.

This study provides an overview of the development of Mapillary data within its first year, including the number of individual contributors and data growth. Mapillary data has already been collected for all five continents, with Europe showing the most contributions. The geographic focus of this study is the 40 largest cities in Germany, and the 4 largest cities in Austria. Since half of the analyzed German cities offer the Google Street View service, which provides the same type of data as Mapillary, we can use these cities to analyse the potential effect of the presence of Google Street View data on the completeness of Mapillary data. Google Street View dates back to 2007 and is currently the most prominent provider of street level photographs. It allows users to access $360^{\circ}$ panorama images from selected roads all over the world.

Contributors to Mapillay can either upload photos through an application on a GPS enabled smartphone, or manually via the Mapillary Website as long as the photograph comes with 
an EXIF file containing geographic coordinates. Google Street View focuses on main roads, since this facilitates fast data capture from cars, and therefore frequent data updates. Google also developed solutions to capture Street View photos for off-road trails, including a special bike, or even a backpack. Mapillary contributors typically use mobile phones to upload data from wherever they are traveling. This frequently also includes off-road paths, such as trails in parks.

Fig. 1(a) provides a screenshot of how a collected street level picture is shown on the Mapillary Website. The lines visualize GPS tracks, along which pictures were taken, and the icon with the arrow indicates the image location together with the orientation of the camera. This picture was taken from a scenic viewpoint at an off-road location in Salzburg, Austria.

\section{Previous Work}

Previous studies report on the use of Google Street View imagery as an effective method for neighbourhood audit to eliminate in-person fieldwork (CLARKE et al. 2010, RUNDLE et al. 2011, GRIEW et al. 2013, VANWOLLEGHEM et al. 2014). Google Street View imagery has also been analysed using computer vision to determine the geographic position of other photos, or to identify road features (ZAMIR \& SHAH 2010, HARA et al. 2013). Previous research has, however, not analysed the spatial coverage and completeness of Google Street View. This will be addressed in our paper, at least for selected cities, through assessing the overlap of Google Street View geometries with OpenStreetMap (OSM) road features. One related study compared the completeness of Google Maps road feature data (not Google Street View, though) with those from Bing Maps and OSM for five cities in Ireland, concluding that none of these datasets were substantially better than another (CIPELUCH et al. 2010).

For part of the research that assesses the completeness of Mapillary and Google Street View data in this study, OSM network data is used as a reference dataset. Although OSM is not governed by an authoritative agency that guarantees certain quality standards, OSM was found to provide good coverage for roads in analysed urban areas (GIRRES \& TOUYA 2010, HAKLAY 2010, ZIELSTRA \& ZIPF 2010). OSM coverage of road segments for non-motorized traffic (e.g., walking or cycling trails) was also shown to be of high quality. For example, for seven cities in the US and Europe it was found that the length of off-road bicycle trails mapped in OSM nearly doubled between 2009 and 2013, except in London, or grew even more (HochMAIR et al. 2015). Another study showed that the total length of mapped footpaths was higher in OSM than for a proprietary data provider (TeleAtlas back then, now TomTom) (ZIELSTRA \& HochMAIR 2011) for selected cities in Germany. For example, in Berlin OSM had 3.2 times as many pedestrian related network data as Tele Atlas. For Munich this ratio was even 5.6. These numbers give evidence that OSM provides a robust reference dataset for determining Mapillary and Google Street View data completeness, also for off-road data analysis. 


\section{Study Design}

\subsection{Study Area}

Only the largest 20 German cities provide Google Street View coverage, in addition to some smaller towns, such as Oberstaufen. For our analysis we selected the 40 largest cities in Germany, which includes 20 cities with and 20 cities without Google Street View imagery. This split allows us to study the potential effect of the availability of Street View imagery on Mapillary coverage in different cities. Furthermore, we also examined the Mapillary coverage in the four largest Austrian cities. The effect of Google Street View imagery on Mapillary coverage could not be assessed for Austrian cities since this Google service is not available in Austria. The German and Austrian cities comprising the study area are shown in Fig. 1(b) (green and blue areas). Red lines indicate Mapillary coverage outside the selected cities. As can be seen, the data collection efforts of the Mapillary community have so far focused on urban areas.

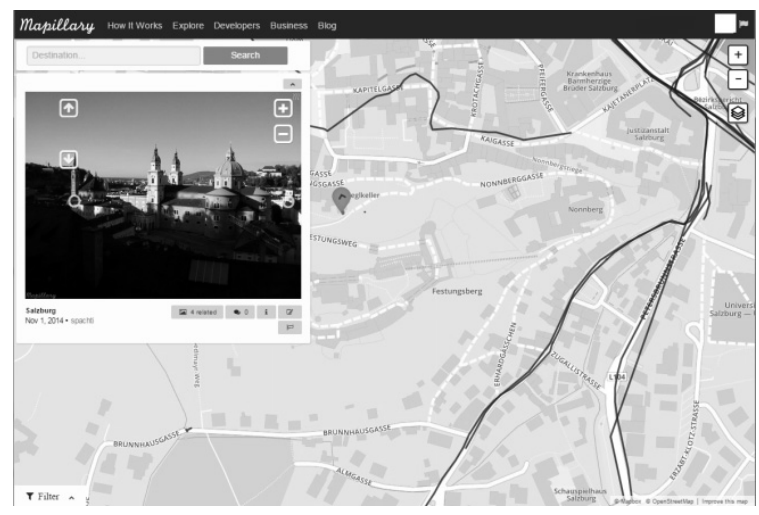

(a)

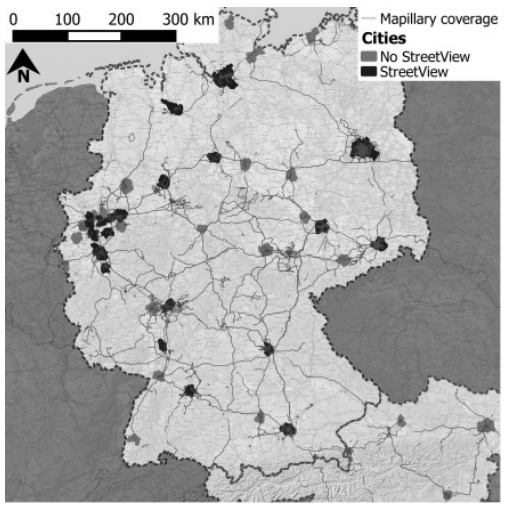

(b)

Fig. 1: Street level image as shown on the Mapillary Website (a) and study area (b)

\subsection{Data Preparation}

\subsubsection{Tile System}

The completeness analysis of this study is based on raster tiles. To facilitate the comparison of Google Street View data with other datasets, we adhered to the tile specifications used in Google Street View. Tiles of the Street View coverage are accessible via the Google Maps API and provided as $256 \times 256$ pixel PNG images. In this system, the world is divided into tiles corresponding to zoom levels. In each zoom level, tiles are indexed by X (column) and $\mathrm{Y}$ (row) values starting from the top-left corner. Zoom level 0 covers the whole world in one tile. The number of tiles in each zoom level is $2^{2 z}$, where $z$ is the zoom level. Logically, this system can be considered a hierarchy of folders and files, where each zoom level is a folder, each X coordinate is a subfolder and each Y coordinate is a PNG image file. This so called XYZ tile scheme provided by Google became a de facto standard in Web mapping, and is used by other map providers including Bing Maps, Yahoo Maps, OSM, and Mapbox. 
Using this schema allows one to convert geographic coordinates to tile coordinates and the other way round. As a preliminary step in our analysis, we converted all data sources into this schema.

\subsubsection{Google Street View}

Google does not provide information on how its Street View tiles are generated. For this study, zoom level 13 tiles were chosen for the analysis, but Street View coverage tiles are highly generalized by default (Fig. 2a) at that level. To make them comparable to the Mapillary dataset, tiles were regenerated (Fig. 2b). To do so, a vector version of Street View lines was extracted based on the Street View coverage, which was downloaded at zoom level 17 (i.e. further zoomed in), using a client-side script in September 2014. As a result of this step, all PNG tiles appeared in the Web browser's cache. Individual images of zoom level 17 were then extracted from the cache and stored within the XYZ tile folder structure. This zoom level corresponds to a ground pixel size of approximately $1.2 \mathrm{~m}$. After geocoding each tile, the PNG raster tiles were loaded into GRASS GIS, where they were patched together, a thinning algorithm was applied, and the 1 pixel wide raster images were vectorised. All extracted lines were then uploaded to a PostgreSQL database with line geometries.

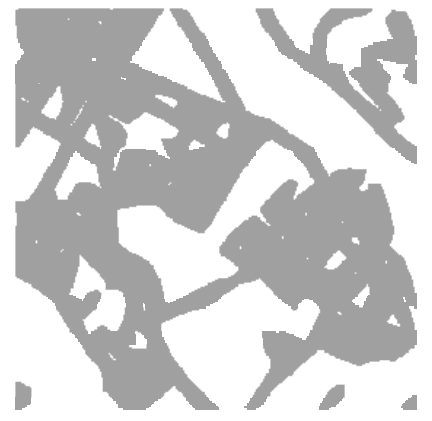

(a)

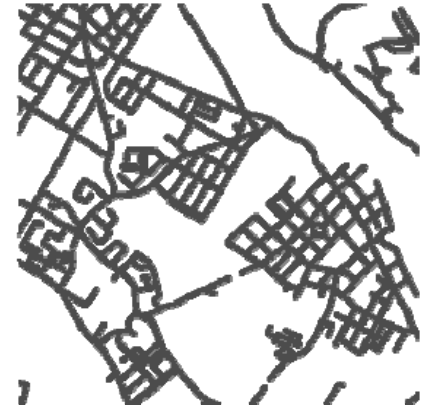

(b)
Fig. 2:

Original (a) and regenerated (b) Street View tiles at zoom level 13

As the last step, TileMill and Mapnik toolkits were used to render tiles at the zoom level 13. Background pixels had a value of 0 , and the pixel size in this zoom level is $\sim 19 \mathrm{~m}$, concealing GPS positioning errors occurring during the data collection process.

\subsubsection{Mapillary}

Mapillary offers various methods to download data via their JSON API. For this study, image sequences were used, which are LineStrings of coherent images and their attributes. Images are taken one after another by walking, driving, or riding a bike. Although geometries and additional attributes of each sequence can be downloaded, the Mapillary team provided us with a database dump that included some additional information, including user ID, timestamp, and geometry of each sequence. The lines are GPS trajectories, where each node is the position of an image. Individual GPS trajectories are mapped as separate lines even if they were taken on the same road. Using map tiles with a zoom level of 13 (pixel size $\sim 19 \mathrm{~m}$ ) was found to be an efficient method to avoid double counting sequences on the same road. This means that segments of multiple lines are counted as only one line if they 
fall within the same pixel. Mapillary tiles were then rendered with the same parameters used to render Street View tiles. We used a dataset that contains LineStrings of photos uploaded to Mapillary up until November 18, 2014.

\subsubsection{OpenStreetMap}

An OSM database dump was downloaded from Geofabrik in October, 2014. All roads were extracted with Osmosis using a highway=[key] filter, and uploaded into a spatially-enabled PostgreSQL database. Since this study uses certain OSM road categories, additional queries were formulated to extract the following road categories:

- Main roads: connect settlements and cities

- Residential roads: minor, lower level roads with moderate traffic

- Pedestrian/Cycle roads: minor elements of the road network used for pedestrians or cyclists for daily routine or recreational purposes

Inaccessible roads, sidewalks, road crossings, tunnels, and indoor features were excluded based on their tags. Further, all pedestrian and bicycle highway features within $25 \mathrm{~m}$ from main and residential roads were removed to be able to assess completeness of off-road pedestrian and bicycle features in Mapillary and Google Street View. In a final step, OSM map tiles were generated for zoom level 13 in the same way as previously done for Google Street View and Mapillary data.

\subsection{Determination of Relative Completeness Between Data Sources}

\subsubsection{Mapillary vs. Google Street View}

20 out of the 40 analysed cities in Germany provide Google Street View service, which allows a comparison between Mapillary and Street View coverage. Since Mapillary is a relatively new service, it can be expected that Street View has better coverage in most analysed areas. However, since Street View is mostly bound to car accessible roads, it can also be expected that Mapillary exceeds Street View coverage in some off-road areas, e.g. at recreational sites inaccessible to cars.

A self-developed python script compared tiles from Mapillary and Street View datasets within predefined boundaries. The script loaded each tile from the two datasets and counted non-zero value pixels within the tile. As a result, each unique tile identifier could be associated with the count values of Mapillary and Street View pixels of that tile. The geographic extent of tiles at zoom level 13 is $\sim 4.9 \times 4.9 \mathrm{~km}^{2}$. Each tile was subsequently divided into 16 squares with a spatial resolution of $\sim 1.2 \times 1.2 \mathrm{~km}^{2}$. This provided enough detail to illustrate local differences in mapping completeness at the city level. This new reference system is also identical to the tile system at zoom level 15. Results were uploaded into a PostgreSQL database with polygon vector geometries. A relative completeness difference that ranges between -1 and 1 was calculated (Equation 1a). A value of 1 indicates that a tile contains only Street View coverage but no Mapillary data, whereas -1 means the opposite.

\subsubsection{Completeness Relative to OpenStreetMap}

The method to calculate Mapillary and Street View completeness relative to predefined OSM road categories is similar. The difference is how pixels are counted. Instead of counting all pixels within a tile, completeness requires to identify only those Mapillary and 
Street View pixels that overlap with pixels of selected OSM road categories. Since the reference tile system is the same for all datasets, it is sufficient to identify the position of OSM road pixels in a tile, and then check whether that specific pixel has a value of one in the other datasets or not. Results were again uploaded to a PostgreSQL database. Overall completeness of each city can then be calculated by selecting tiles within the city boundary and computing the fraction of Mapillary or Street View pixels overlapping with OSM road pixels in the selected tiles (Equation 1b). Calculations were performed on all predefined OSM road categories for Mapillary and Street View.

$d(S V, M a p)=\left\{\begin{array}{ll}0, & \text { if } S V+M a p=0 \\ \frac{(S V-M a p)}{(S V+M a p)}, & \text { if } S V+M a p>0\end{array} \quad C_{\mathrm{r}}=\frac{\sum P x_{\mathrm{r}}}{\sum O S M_{\mathrm{r}}}\right.$

Where $d$ is the relative completeness difference, $S V$ is the count of Street View pixels and Map is the count of Mapillary pixels

Where $C_{r}$ is the completeness of Mapillary or Street View on a road category, $P x_{r}$ is a pixel overlapping with a pixel from the OSM road category $r$ and $O S M_{r}$ is a pixel of the OSM road category $r$.

(b)

Equation 1: Relative completeness difference (a) and computation of completeness (b)

\section{Results}

\subsection{User Contributions}

Fig. 3(a) shows the number of Mapillary users actively contributing in Germany and Austria for each month. The majority of users contribute on a regular basis (returning users). Until November 2014 the total number of contributing users reached 388. Mappers covered sequences of more than $46500 \mathrm{~km}$, whereby it must be noted that some of the sequences were taken on the same roads. Fig. 3(b) shows the distribution of data contributors for various total distance ranges. The most active contributor uploaded pictures along almost 6100 $\mathrm{km}$ to Mapillary. Fig. 3(b) also expresses inequalities in data contributions among volunteers, which has been previously identified for other VGI data sources, such as OSM (NEIS \& ZIELSTRA 2014) or drone images (HochMAIR \& ZIELSTRA 2014). In those VGI data sources, but also with Mapillary, few users contribute most of the data, whereas the majority of users make only few data contributions. In the case of Mapillary, more than $60 \%$ (205) of the users contributed less than $10 \mathrm{~km}$. Conversely, only 5\% (19) mapped $500 \mathrm{~km}$ or more. Another aspect of the contribution pattern is the number of cities a user mapped in. Fig. 3(c) shows that a large majority of users $(40 \%)$ contributed in only one city, as opposed to only $4 \%$ of users who contributed in 5 or more study cities, which again reflects contribution inequality. 

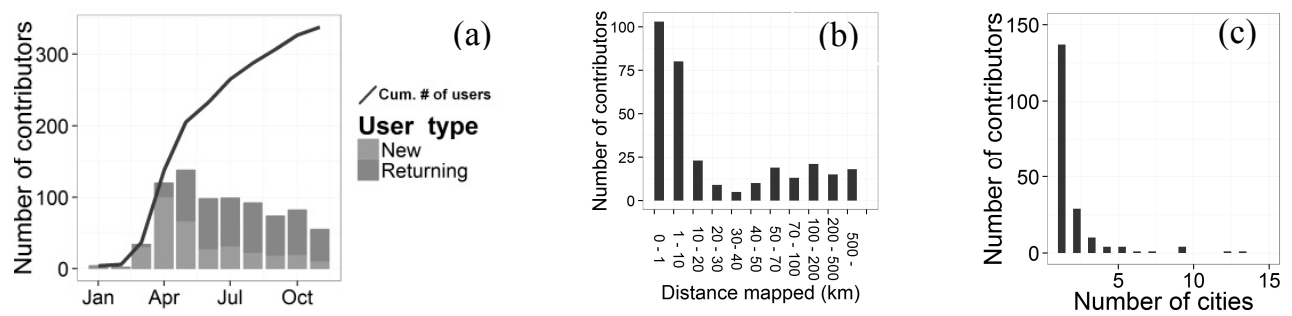

Fig. 3: User contributions to Mapillary

\subsection{Completeness}

\subsubsection{Relative Completeness Difference Between Mapillary and Street View}

The relative completeness difference between Mapillary and Google Street View data was determined for the 20 largest cities in Germany. This computation was not possible for Austria, where Street View is not provided. First, all tiles within a city were selected, then all relevant pixels within those tiles were used to calculate the relative completeness difference based on Equation 1a. Values range between 0.38 (Dresden) and 0.98 (Düsseldorf). The values are all positive, meaning that Google Street View provides better overall coverage in the analysed cities.

The median of relative completeness differences is 0.84 . An outlier (Dresden) with a value of 0.38 indicates that this city has high Mapillary coverage compared to other cities. Fig. 4(a) and 4(b) visualize patterns of relative completeness difference for Dresden and Berlin (the latter with a value of 0.65 ). Purple tiles (without borders) show areas where more roads
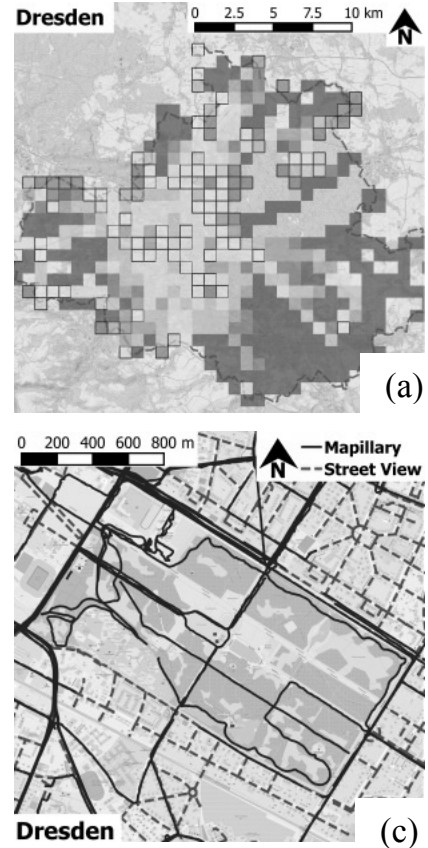

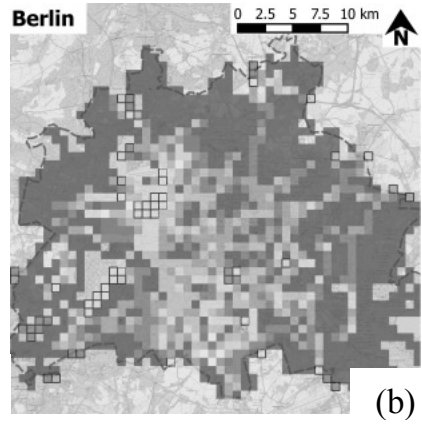

(b)

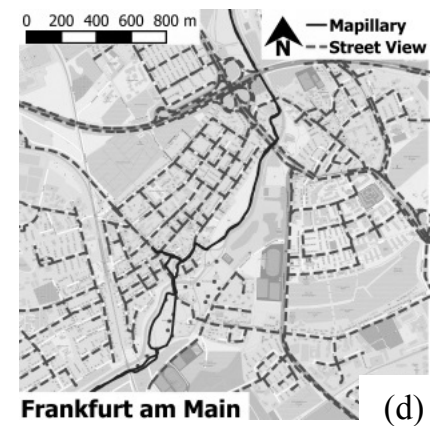

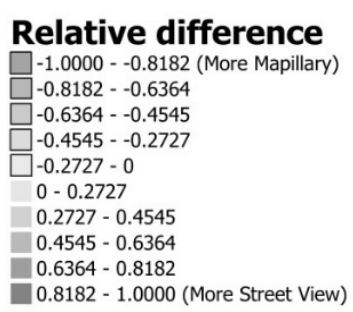

Fig. 4:

Spatial distribution of relative completeness difference (a-b) and examples of better Mapillary coverage (c-d) 
are mapped in Google Street View, whereas orange tiles (with borders) show areas with higher Mapillary coverage. The low relative completeness difference for Dresden matches the visual appearance of the tile map with its large portions of orange areas in the suburbs and the town centre. In contrast, Berlin shows fewer orange areas. Whereas Street View imagery mostly relies on the road network, Mapillary photos are often taken off-road or on minor roads. Typical examples can be found on riversides. Mapillary is more complete in a park in Dresden (Fig. 4(c)) or along a river in Frankfurt (Fig. 4(d)). In general, differences between both data sources are smaller in city centres or other populated areas, indicated through lighter colours.

\subsubsection{Completeness Relative to OpenStreetMap Features}

At the city level, Street View is more complete than Mapillary, but it is limited to the 20 biggest German cities, with some exceptions. Completeness values relative to OSM are shown in Table 1 for some selected German cities and the largest four cities in Austria.

Table 1: Completeness relative to selected OSM road categories (\%)

\begin{tabular}{|c|c|c|c|c|c|c|c|c|}
\hline & \multirow[t]{2}{*}{ City } & \multicolumn{2}{|c|}{ OSM Main } & \multicolumn{2}{|c|}{ OSM Residential } & \multicolumn{2}{|c|}{ OSM Ped./Cycle } & \multirow{2}{*}{$\begin{array}{l}\text { Street } \\
\text { View }\end{array}$} \\
\hline & & Google & Mapillary & Google & Mapillary & Google & Mapillary & \\
\hline \multirow{4}{*}{ } & Vienna & 0 & 13.93 & 0 & 2.26 & 0 & 0.80 & \\
\hline & Graz & 0 & 32.72 & 0 & 16.25 & 0 & 7.00 & \\
\hline & $\operatorname{Linz}$ & 0 & 8.82 & 0 & 2.39 & 0 & 0.82 & \\
\hline & Salzburg & 0 & 67.73 & 0 & 13.47 & 0 & 5.68 & \\
\hline \multirow{11}{*}{ 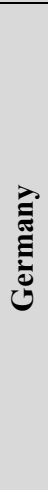 } & Berlin & 91.44 & 42.41 & 81.44 & 8.10 & 1.67 & 1.86 & $\mathrm{X}$ \\
\hline & Hamburg & 83.35 & 15.36 & 72.80 & 1.84 & 2.15 & 0.54 & $\mathrm{X}$ \\
\hline & Munich & 90.08 & 22.11 & 82.81 & 3.76 & 2.43 & 1.52 & $\mathrm{X}$ \\
\hline & Frankfurt & 82.72 & 14.95 & 63.66 & 1.37 & 2.62 & 3.63 & $\mathrm{X}$ \\
\hline & Düsseldorf & 87.97 & 1.32 & 74.44 & 0.19 & 3.30 & 0.19 & $\mathrm{X}$ \\
\hline & Dortmund & 85.72 & 20.82 & 79.02 & 2.51 & 3.76 & 0.65 & $\mathrm{X}$ \\
\hline & Bremen & 85.33 & 6.46 & 75.49 & 0.45 & 1.85 & 0.28 & $\mathrm{X}$ \\
\hline & Dresden & 86.77 & 59.01 & 78.10 & 18.75 & 2.18 & 4.65 & $X$ \\
\hline & Nuremberg & 92.10 & 22.94 & 80.19 & 3.21 & 2.96 & 0.54 & $X$ \\
\hline & Duisburg & 88.84 & 4.31 & 83.99 & 0.07 & 3.49 & 0.12 & $X$ \\
\hline & Bielefeld & 77.11 & 20.50 & 58.23 & 1.50 & 1.72 & 0.67 & $X$ \\
\hline
\end{tabular}

Major roads are well covered in Google Street View, with completeness ranging between $77 \%$ (Bielefeld) and 92\% (Nuremberg). Numbers are also high for residential roads and ranging between $84 \%$ (Duisburg) and 58\% (Bielefeld), but much smaller for off-road pedestrian/cycle paths with a maximum of $4 \%$. Completeness is considerably smaller for Mapillary in all categories, with a few exceptions in the pedestrian/cycle category. For the latter category it is possible that some of the positive percentage numbers in Google Street View stem from images taken by cars along roads in the vicinity of pedestrian or cycling paths. This situation can occur near roads that were not previously excluded from the analysis (only main or residential roads were removed), but are still driveable, such as "track" or "service" roads in OSM. Salzburg provides the most complete coverage of main roads in 
Mapillary among all cities with a value of $68 \%$. Considering all 44 selected cities, main roads in Mapillary are mapped most completely $(18.6 \%)$, followed by residential roads $(3.2 \%)$ and pedestrian or cycling paths $(1.1 \%)$.

No association could be identified between city size and data completeness in Mapillary. For example, some of the larger cities, such as Düsseldorf (pop. 590,000), Bremen (pop. $\sim 550,000$ ), or Duisburg (pop. $\sim 490,000$ ) are poorly covered in Mapillary. Previous research suggests that the availability of freely available datasets from other sources in a region diminishes the community's motivation for voluntary data collection efforts, e.g. in the case of OSM (ZIELSTRA \& HOCHMAIR 2011). However, mean comparison of Mapillary completeness between cities with and without Google Street View did not reveal statistically significant differences in Mapillary completeness between both groups of cities.

\subsubsection{Data Growth}

Fig. 5(a) shows the growth of average Mapillary completeness values for the three OSM road categories and the selected 44 cities. Main roads are growing more rapidly than other road categories. Fig. 5(b)-(d) show the completeness growth curves for five selected cities in the three road categories. Dresden and Salzburg reveal the highest current completeness in all three categories. A very active mapping period can be identified between May and July 2014 in Salzburg. For the other four selected cities, growth slowed down in August, 2014. However, this trend cannot be observed when considering all 44 selected cities (Fig. 5(a)).
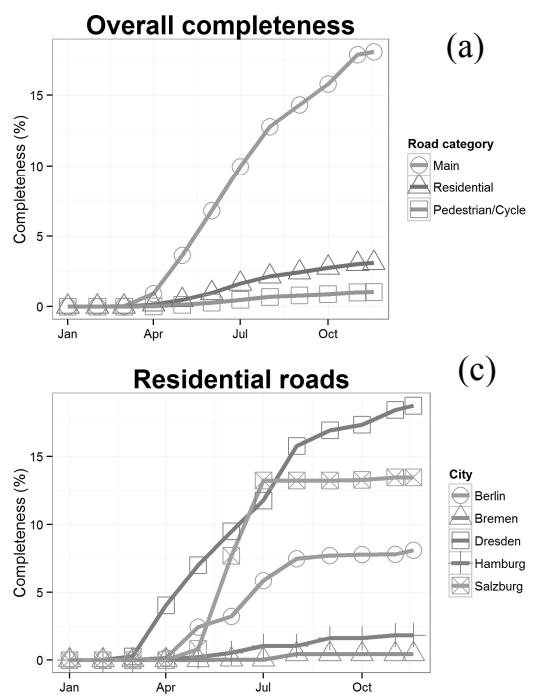

(a)
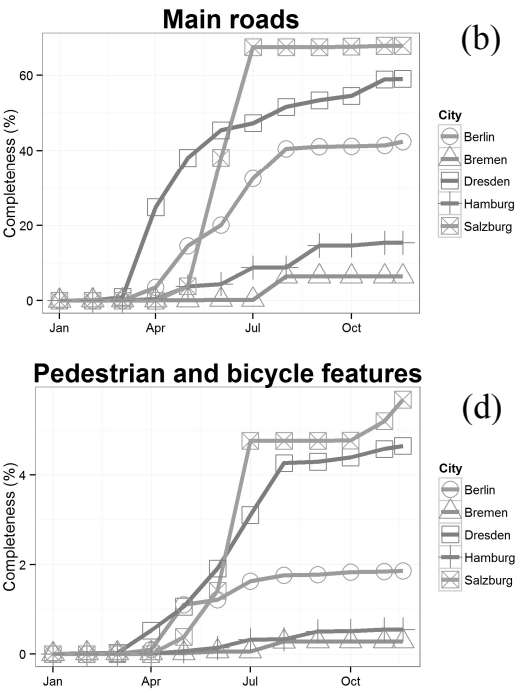

(b)

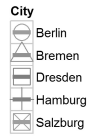

d)
Fig. 5: Completeness growth in Mapillary data: Average for three road categories in 44 cities (a), and completeness in five selected cities for three road categories (b)-(d) 


\section{Conclusion and Future Work}

This study analysed the completeness and development of Mapillary data, which presents an alternative data source of street level photographs to commercial datasets. This study focused on 40 German and 4 Austrian cities. A comparison with Google Street View showed that Google Street View provides a better coverage than Mapillary, with a few exceptions. Since Mapillary is not bound to professional equipment that needs to be moved by car, it could become a complimentary data source of street level imagery to Google Street View for footpaths and bicycle trails, even in those cities where Google Street View is present.

For future work we plan to extend the spatio-temporal analysis of Mapillary data beyond Germany and Austria. It is yet to be seen if privacy concerns of local residents will hamper the continuous growth of Mapillary, as was the case for Google Street View in some European countries. Another aspect of future work is to analyse to which extent Mapillary is used as a data source for OSM. Tags in the source field of OSM features indicate that OSM mappers are already using Mapillary imagery to add landmark point features, such as a bus stop, which can be visually identified on Mapillary street level photographs. Germany was also one of the most active regions during the beginning of the OSM project, similar to Mapillary. We therefore plan to analyse whether the same group of users that pushed the OSM project is also actively contributing to the Mapillary project, or whether Mapillary reaches out to a new crowd of voluntary mappers.

\section{References}

Clarke, P., Ailshire, J., Melendez, R., Bader, M. \& Morenoff, J. (2010), Using Google Earth to conduct a neighborhood audit: Reliability of a virtual audit instrument. Health \& Place, 16 (6), 1224-1229.

GirReS, J. F. \& TOUYA, G. (2010), Quality assessment of the French OpenStreetMap dataset. Transactions in GIS, 14 (4), 435-459.

GoodchILD, M. F. (2007), Citizens as Voluntary Sensors: Spatial Data Infrastructure in the World of Web 2.0 (Editorial). International Journal of Spatial Data Infrastructures Research (IJSDIR), 2, 24-32.

Griew, P., Hillsdon, M., Foster, C., Coombes, E., Jones, A. \& Wilkinson, P. (2013), Developing and testing a street audit tool using Google Street View to measure environmental supportiveness for physical activity. International Journal of Behavioral Nutrition and Physical Activity, 10 (1), 103.

HAKLAY, M. (2010), How good is Volunteered Geographical Information? A comparative study of OpenStreetMap and Ordnance Survey datasets. Environment and Planning B: Planning and Design, 37 (4), 682-703.

Hara, K., Le, V., Sun, J., Jacobs, D. \& Froehlich, J. E. (2013), Exploring Early Solutions for Automatically Identifying Inaccessible Sidewalks in the Physical World Using Google Street View. Human Computer Interaction Consortium 2013.

Hochmair, H. H. \& Zielstra, D. (2014), Analysing User Contribution Patterns of Drone Pictures to the dronestagram Photo Sharing Portal. Journal of Spatial Science. 
Hochmair, H. H., Zielstra, D. \& NeIs, P. (2015), Assessing the Completeness of Bicycle Trail and Designated Lane Features in OpenStreetMap for the United States. Transactions in GIS, 19 (1), 63-81.

NeIS, P. \& ZIELSTRA, D. (2014), Recent developments and future trends in volunteered geographic information research: The case of OpenStreetMap. Future Internet, 6 (1), $76-$ 106.

Rundle, A. G., Bader, M. D. M., Richards, C. A., Neckerman, K. M. \& Teitler, J. O. (2011), Using Google Street View to Audit Neighborhood Environments. American Journal of Preventive Medicine, 40 (1), 94-100.

Vanwolleghem, G., Dyck, D. V., Ducheyne, F., Bourdeaudhuij, I. D. \& Cardon, G. (2014), Assessing the environmental characteristics of cycling routes to school: a study on the reliability and validity of a Google Street View-based audit. International Journal of Health Geographics, 13 (19).

ZAMIR, A. R. \& SHAH, M. (2010), Accurate Image Localization Based on Google Maps Street View. In: Danillidis, K., Maragos. P. \& Paragios, N. (Eds.), Computer Vision - ECCV 2010. Springer, Berlin/Heidelberg, 255-268.

ZIElstRA, D. \& HochmaIR, H. H. (2011), A Comparative Study of Pedestrian Accessibility to Transit Stations Using Free and Proprietary Network Data. Transportation Research Record: Journal of the Transportation Research Board, 2217, 145-152. 